\title{
A Rare Case of Bilateral Optic Neuritis and Guillain-Barré Syndrome Post Mycoplasma pneumoniae Infection
}

\author{
Aravindhan Baheerathan ${ }^{\mathrm{a}}$, Amy Ross Russella, Fion Bremner ${ }^{\mathrm{b}}$, and Simon F. Farmer ${ }^{\mathrm{a}}$ \\ aDepartment of Neurology, National Hospital for Neurology and Neurosurgery, London, United Kingdom; 'bepartment of Neuro- \\ ophthalmology, National Hospital for Neurology and Neurosurgery, London, United Kingdom
}

\section{ABSTRACT}

Neurological complications are the most commonly encountered extra-pulmonary manifestation of infection with Mycoplasma pneumoniae (M. pneumoniae). Here the authors report the case of a 39-yearold woman who was admitted with acute-onset bilateral visual loss coinciding with ascending numbness. Clinical examination, neurological imaging, and nerve conduction studies revealed a syndrome of bilateral optic neuritis and Guillain-Barré syndrome (GBS). Serological testing confirmed recent exposure to M. pneumoniae. The patient did not experience any clinical benefit with pulsed intravenous methylprednisolone but demonstrated marked clinical and radiological improvement following 5 days of plasma exchange. This report will explore the diagnostic and therapeutic approach to patients with neuro-ophthalmological and neurological complications of $M$. pneumoniae infection in addition to discussing previously encountered cases.
ARTICLE HISTORY

Received 15 June 2016

Revised 13 September 2016

Accepted 14 September 2016

\section{KEYWORDS}

Mycoplasma pneumoniae; post-infectious inflammatory neuropathy; post-infectious optic neuropathy; plasma exchange; optic neuritis; Guillain-Barré syndrome

\section{Case report}

A 39-year-old Afro-Caribbean woman was admitted to our neurological department from the local ophthalmic hospital casualty department, having presented with acute-onset bilateral visual loss.

Fifteen days prior to presentation, she had experienced a dry cough with flu-like symptoms, predominantly myalgia, in association with a dull headache. The patient had been prescribed 5 days of amoxicillin in primary care. These symptoms had resolved, but were followed 5 days later by bilateral eye pain, pain on eye movements, and a reduction of vision in each eye over the subsequent 5 days. She also described pins and needles in her fingers and toes, gradually progressing over the same time course.

Upon initial assessment she reported no perception of light in either eye. Pupillary reflexes were absent. She had bilateral swelling of the optic discs with splinter haemorrhages (Figure 1a). There was bilateral globe tenderness. The remainder of the cranial nerve examination was normal.

Examination of the arms demonstrated normal tone and full power. She had reduced light touch and pin prick to the level of the wrists. Reflexes were present and symmetrical. Examination of the legs showed normal tone and full power. Light touch and pin prick sensation was reduced to the ankles. Reflexes were present on day 1 , but ankle-knee, supinator, and bicep reflexes had become absent by day 4. Plantar reflexes were flexor bilaterally.

Magnetic resonance imaging (MRI) of the brain and orbits demonstrated enlargement of both optic nerves with gadolinium enhancement (Figure 2a). The reminder of the brain parenchyma was normal with no evidence of leptomeningeal enhancement. Positron emission tomography-computed tomography (PET-CT) of the whole body did not show any pathological uptake. Chest X-ray was unremarkable.

Electrophysiological investigation demonstrated a motor-predominant acquired distal demyelinating polyneuropathy, consistent with Guillain-Barré syndrome (GBS).

Lumbar puncture gave an opening pressure of $16 \mathrm{~cm} \mathrm{H}_{2} \mathrm{O}$. Cerebrospinal fluid (CSF) showed protein of $0.21 \mathrm{~g} / \mathrm{L}$, white cell count of 4 , and negative culture and cytology. Full blood count, electrolytes, and renal function and liver

CONTACT Amy Ross Russell amyrossrussell@nhs.net $\mathrm{B}$ Neurology SpR, Wessex Neurology Centre, Southampton General Hospital, Tremona Road, S016 6YD, UK.

Aravindhan Baheerathan and Amy Ross Russell contributed equally to this paper and are co-first authors.

Color versions of one or more of the figures in the article can be found online at www.tandfonline.com/ioph.

(C) 2017 Taylor \& Francis 

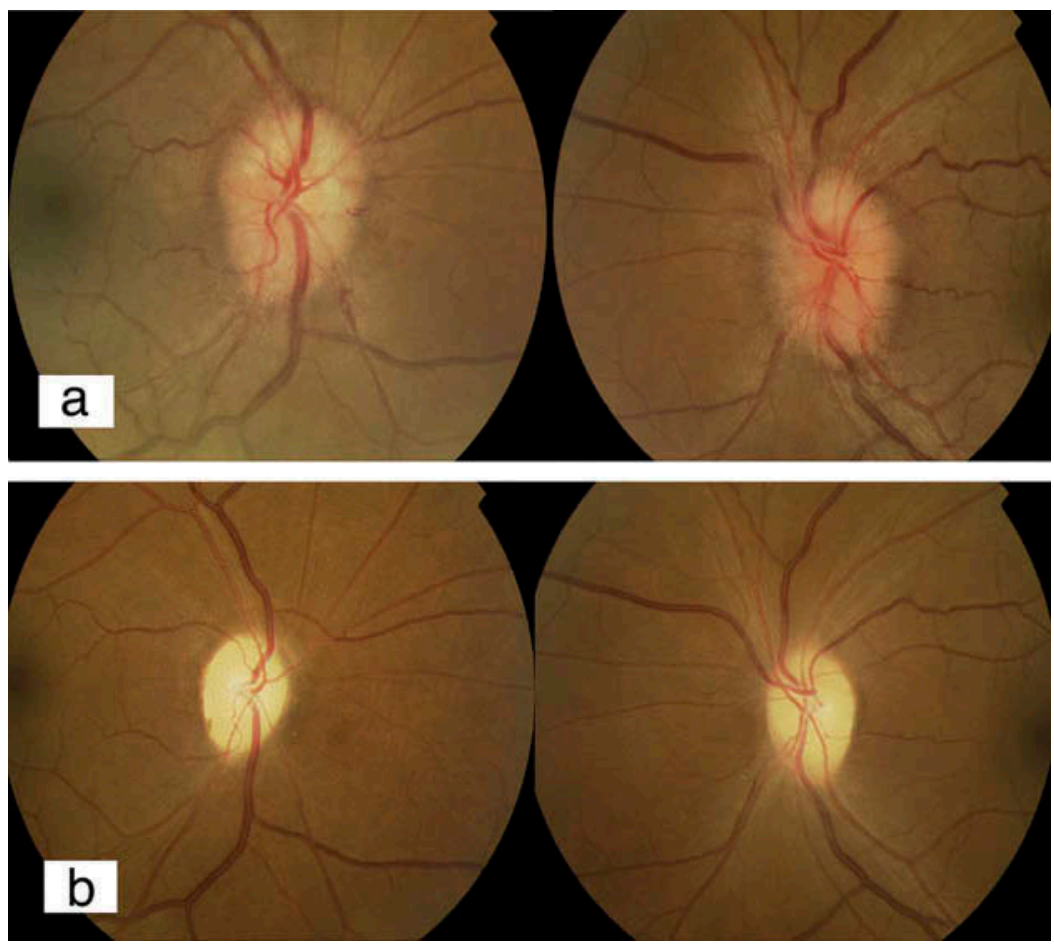

Figure 1. (a) Fundal imaging on admission demonstrating bilateral optic disc swelling with splinter haemorrhages in both right and left eyes. (b) Fundal imaging 2 months following initial treatment demonstrating bilateral optic disc pallor.

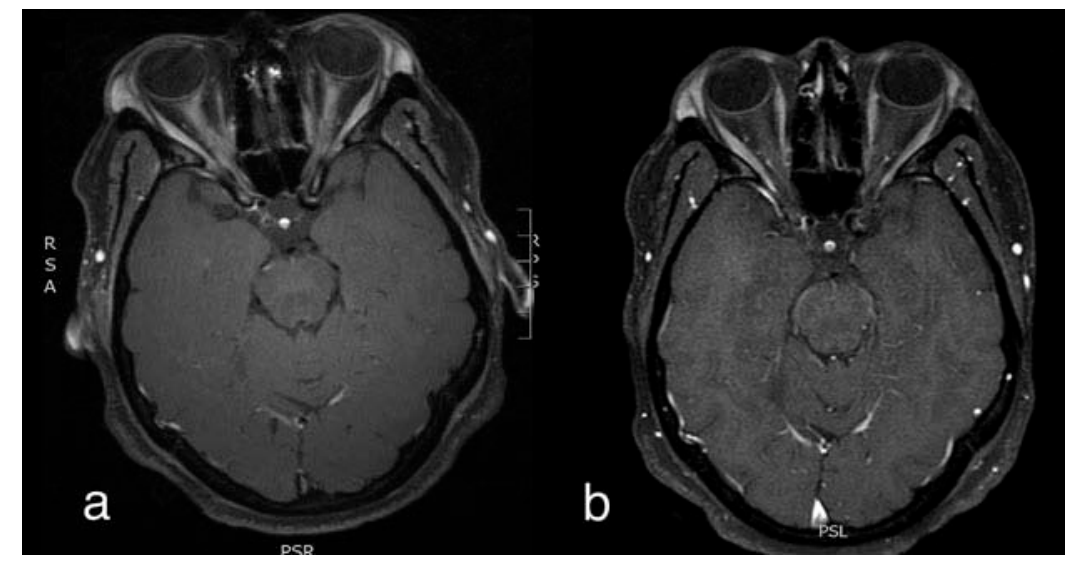

Figure 2. (a) Axial fat-saturated T1-weighted MRI post gadolinium enhancement showing bilateral optic nerve enhancement. (b) Following plasma exchange, marked improvement of the swelling of both optic nerves with minimal enhancement seen post-gadolinium administration.

function tests were normal. Human immunodeficiency virus (HIV), aquaporin-4, anti-neuronal antibodies, and anti-myelin oligodendrocyte glycoprotein (anti-MOG) antibodies were negative. Mycoplasma pneumoniae (M. pneumoniae) serology showed a titre of 1:280 and immunoglobulin $\mathrm{M}$ (IgM) antibodies against $M$. pneumoniae were strongly positive, highly suggestive of acute infection. M. pneumoniae was not isolated from the CSF, and CSF $M$. pneumoniae polymerase chain reaction (PCR) was negative.
A diagnosis of bilateral optic neuritis and GBS secondary to Mycoplasma pneumoniae infection was made.

The patient was treated on admission with six doses of $1 \mathrm{~g}$ intravenous methylprednisolone on consecutive days, which did not result in any change in visual acuity (VA). We proceeded to plasma exchange, completing five courses over 5 days; $40 \mathrm{~mL} / \mathrm{kg}$ of plasma was removed per course, and 5\% human albumin solution was utilised as replacement fluid. This produced rapid clinical improvement of VA, and she also 
reported gradual improvement of her sensory symptoms with complete resolution of her pins and needles. On day 8 (day 3 of plasma exchange), her right eye still had no perception of light but she was able to see the outline of faces in the left eye. Two days later (day 10), she was able to see shapes with her right eye, and her left VA was 6/36.

At day 14, her Goldman perimetry showed generally depressed visual fields (Figure 3a). Upon completion of plasma exchange, her VA was $6 / 24$ on the right and 6/18 on the left.

Upon receipt of the Mycoplasma serology, the patient was commenced on intravenous clarithromycin. At discharge (day 29), the VA in the right eye was $6 / 18$ and the VA in the left eye was 6/9. She was discharged on an oral taper of prednisolone over 1 month starting at $1 \mathrm{mg} / \mathrm{kg}$.

Repeat imaging 2 weeks after completion of plasma exchange demonstrated radiological resolution of the bilateral optic neuritis (Figure 2b), and repeat nerve conduction studies showed marked improvement of the polyneuropathy. Fundal photography 8 weeks post treatment demonstrated bilateral optic disc pallor (Figure 1b).

Eight months later, upon review in the outpatient clinic, the patient had not had any relapse of her neuritis following complete tapering of steroid therapy. Her VA was $6 / 5$ in the left eye and 6/12 in the right eye. Her colour vision was $17 / 17$ on the left and 3/17 on the right using Ishihara plates. There were persisting visual field deficits in the right and left eyes (Figure 3b). At follow-up, her colour vision was assessed using Ishihara colour plates; she scored $17 / 17$ on the left and $3 / 17$ on the right.

\section{Discussion}

Mycoplasma pneumoniae is a commonly encountered pathogen that is often associated with infection of the respiratory system and produces a highly variable clinical phenotype, ranging from a mild upper respiratory tract infection to a severe pneumonia. ${ }^{1}$ In addition to its respiratory manifestations, it can also give

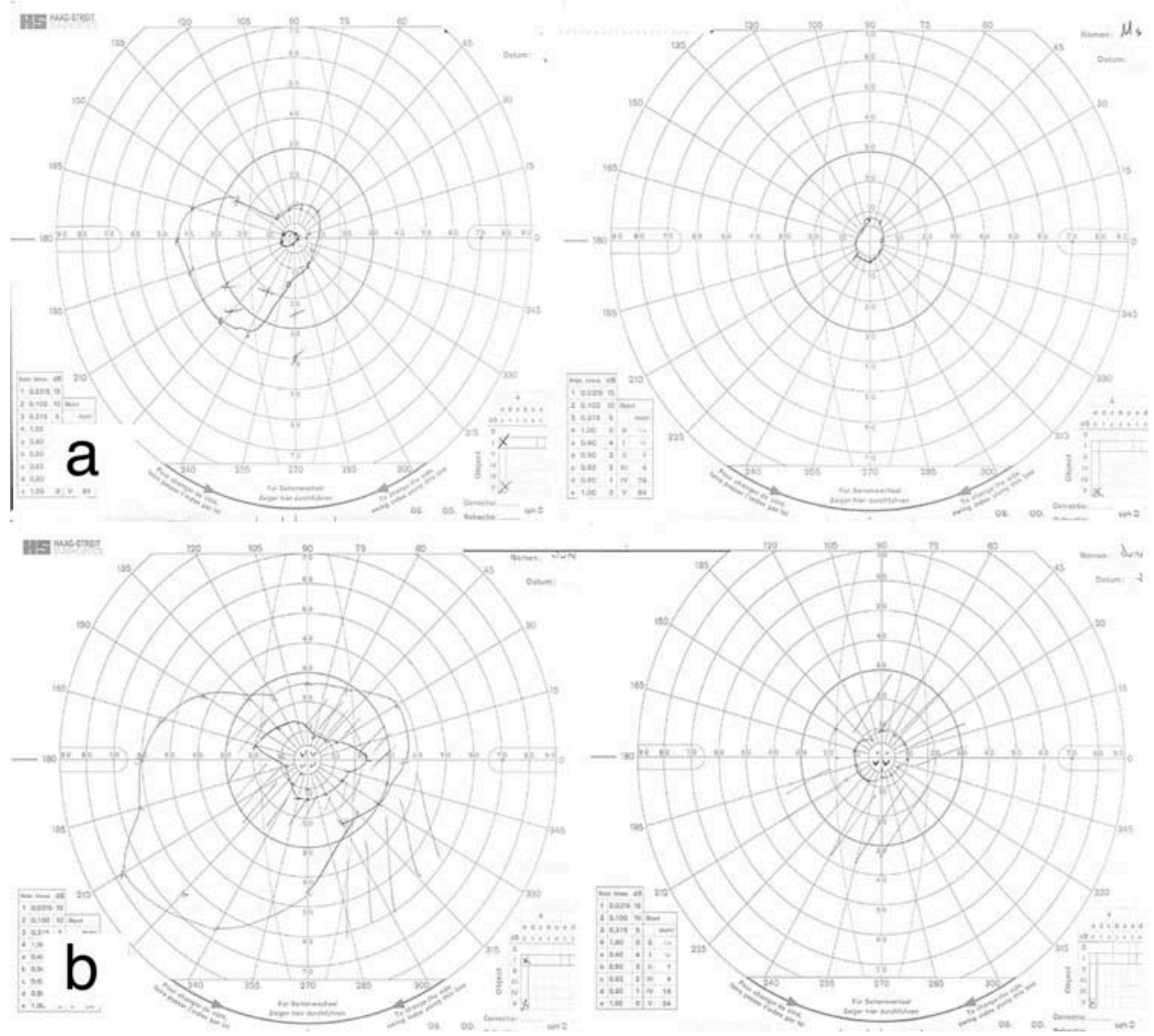

Figure 3. (a) Visual fields obtained via Goldman perimetry at 14 days post-admission demonstrating a globally depressed visual field. (b) Visual fields assessed via Goldman perimetry at 8 months post-completion of plasma exchange, demonstrating some improvement in the visual field. 
Table 1.

\begin{tabular}{l}
\hline Neurological manifestations of Mycoplasma pneumoniae infection \\
\hline CNS \\
Optic neuritis \\
Encephalitis \\
Meningoencephalitis \\
Aseptic meningitis \\
Cerebellar ataxia \\
Choreoathetosis \\
Transverse myelitis \\
Acute psychosis \\
Ischaemic stroke \\
PNS \\
Cranial nerve palsies \\
Guillian Barre syndrome \\
Polyradiculitis \\
Peripheral neuropathy
\end{tabular}

rise to numerous extra-pulmonary manifestations, particularly neurological. $^{1}$

Neurological complications have been reported to occur in between $0.1 \%$ and $7 \%$ of patients infected with Mycoplasma pneumoniae, typically occurring between 2 and 14 days after initial respiratory symptoms. ${ }^{2}$ These have been widely reported in the paediatric population. ${ }^{1-3}$ Central nervous system (CNS) manifestations include optic neuritis, encephalitis, meningoencephalitis, aseptic meningitis, transverse myelitis, cerebellar ataxia, choreoathetosis, ischaemic stroke, and syndrome of inappropriate anti-diuretic hormone. Peripheral nervous system manifestations are less commonly described but include cranial nerve palsies, GBS, polyradiculitis, and peripheral neuropathy (Table 1). ${ }^{1-5}$ Table 2 highlights all cases published (in English) of optic neuritis that has occurred post $M$. pneumoniae infection.

The pathogenesis of the neurological complications of M. pneumoniae infection is poorly understood and is a source of debate. ${ }^{1,17}$ It has been postulated that there may be two distinct mechanisms of $M$. pneumoniae-induced neurological disease: first, the direct result of $M$. pneumoniae invasion into the $\mathrm{CSF}^{18}$ and second, a systemic immune-mediated response to infection, with CNS sequelae. The latter mechanism fits best with the clinical presentation of our patient.

Diagnosis of Mycoplasma pneumoniae is challenging. Historically, cold agglutinins were used to aid the diagnosis of Mycoplasma pneumoniae infection. Cold agglutinins are produced 1-2 weeks after infection in $50 \%$ of patients and may persist for several weeks, but due to their poor sensitivity and specificity, they are now considered obsolete in making a diagnosis. Microbial culture is technically demanding and seldom successful in routine medical practice. Diagnosis of M. pneumoniae infection is primarily achieved through a consistent clinical presentation that coincides with positive serological testing, such as passive agglutination, complement fixation, and enzyme-linked immunosorbent assay (ELISA). Serological tests for anti-M. pneumoniae antibody represent the most common method for retrospective diagnosis of $M$. pneumoniae infections, but they depend on convalescent sera for confirmation and false-positive results from cross-reactivity are a problem. ${ }^{1,2}$ Seroconversion is defined as a 4 -fold increase in titre between acute and convalescent sera, or a single high anti-M. pneumoniae complement fixation antibody titre of $>1: 128 .^{1}$ The latter criteria was met in our patient. A combination of PCR and serology is recommended for accurate diagnosis, especially for patients with neurological and other extra-pulmonary manifestations. ${ }^{1,2}$

The concomitant occurrence of bilateral optic neuritis and GBS post M. pneumoniae infection has been reported on five previous occasions in the published literature; however, in four of the five cases the onset of an GBS preceded the onset of bilateral optic neuritis (ON). Ginestal et al. ${ }^{11}$ described a similar case of bilateral ON preceding GBS; however, in contrast to our case, their patient demonstrated GBS with predominantly motor symptoms. In three of the five cases, excellent motor recovery and partial visual recovery were achieved with the use of immunotherapy (two cases treated with a combination of steroid and intravenous immunoglobulins, one with plasma exchange, one with a combination of plasma exchange and steroids, and one with only intravenous immunoglobulins). Whilst our patient did not improve with the use of intravenous steroid therapy, the use of plasma exchange produced rapid recovery of visual acuity and resolution of the sensory symptoms.

The role of antimicrobials in the treatment of neurological manifestations of $M$. pneumoniae infection remains controversial, whilst the mechanism of disease is unclear. If $M$. pneumoniae infection is directly responsible for the pathology, 


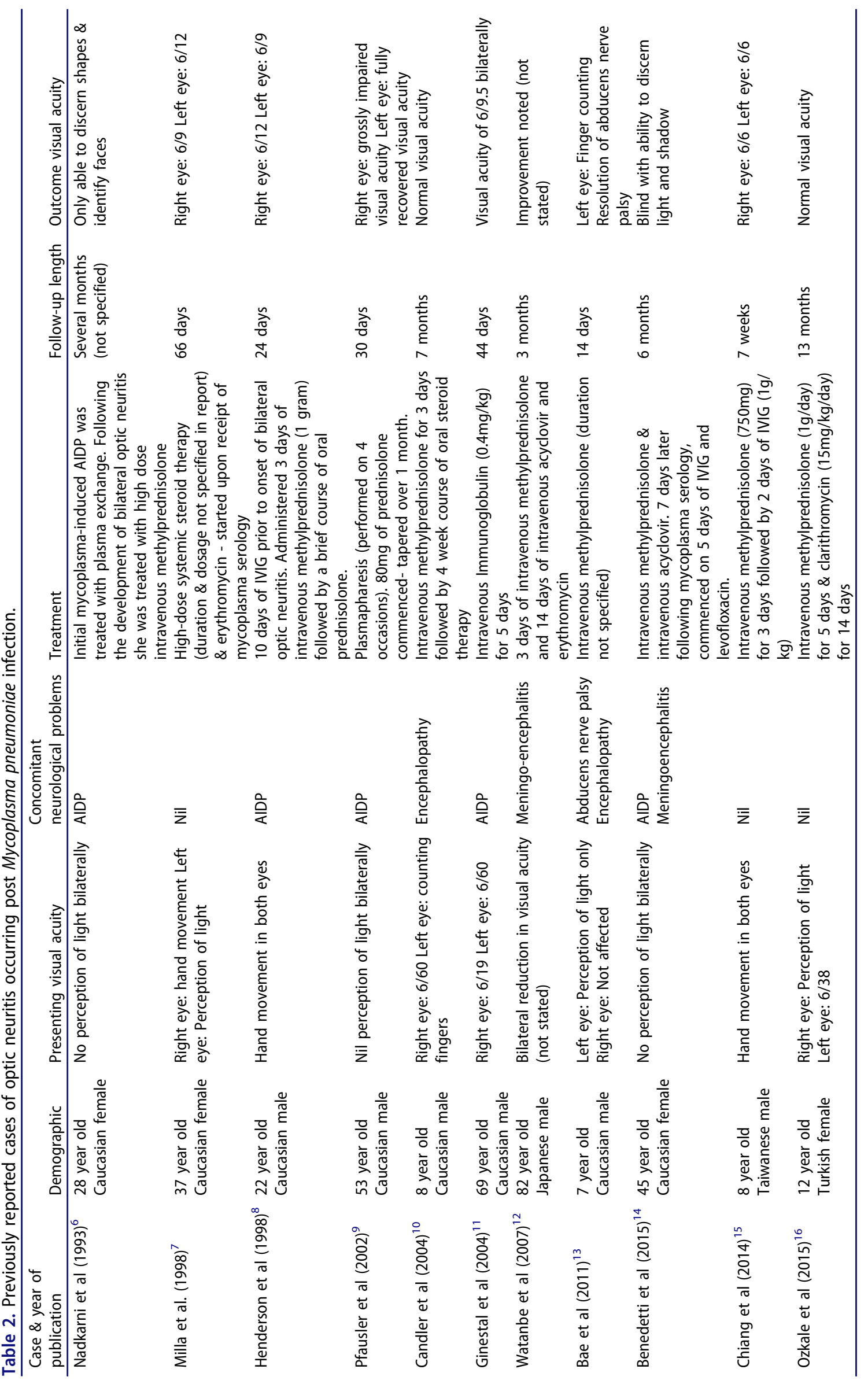


then antibiotic treatment would be recommended. Antibiotic treatment recommendations also need to take into account CSF bioavailability. If the mechanism is immune mediated, then it is less clear whether antimicrobial therapy is appropriate, ${ }^{1,10}$ particularly after the acute illness has resolved. ${ }^{1}$ In practice, patients such as ours are treated urgently with antibiotic and immune therapy.

\section{Conclusion}

We report a rare case of bilateral optic neuritis in association with GBS secondary to Mycoplasma pneumoniae with severe neuro-ophthalmological deterioration and a poor initial response to intravenous steroid therapy, thus requiring escalation to plasma exchange, which showed good response.

The most effective treatment protocol is not known, and good clinical response has been seen with steroids and intravenous immunoglobulin therapy. However, given our patient's dramatic response to plasma exchange in the absence of any improvement with steroid therapy, we believe this should be considered early in patients presenting with this clinical syndrome, in order to minimise the potential for long-term neurological and neuro-ophthalmic damage.

\section{Key messages}

- M. pneumoniae can lead to a diverse range of neurological manifestations and should be considered, particularly in cases with an infectious prodrome.

- Infection can be difficult to diagnose, but serological testing and PCR are the most commonly utilised methods.

- Early immunotherapy and antibiotic therapy are essential.

\section{Acknowledgements}

We would like to thank Najwa Bantan, Farrah Jabeen, Christina Dudau, and Tarek Yousry for their help with radiology interpretation, and Dr. C. Cordivari and Dr. D. Fialho for their help with neurophysiology. S.F.F. acknowledges support from the UCLH Biomedical Research Centre.

\section{Declaration of interest}

The authors report no conflicts of interest. The authors alone are responsible for the content and writing of the article.

\section{References}

[1] Sánchez-Vargas FM, Gómez-Duarte OG. Mycoplasma pneumoniae - an emerging extra-pulmonary pathogen. Clin Microbiol Infect 2008;14:105-117.

[2] Guleira R, Nisar N, Chawla TC, Biswas NR. Mycoplasma pneumoniae and central nervous system complications: a review. J Lab Clin Med 2005146:55-56.

[3] Tsiodras S, Kelesidis I, Kelesidis T, Stamboulis E, Gimarellou H. Central nervous system manifestations of Mycoplasma pneumoniae infections. $J$ Infect 2005;51:343-354.

[4] Yimenicioğlu S, Yakut A, Ekici A, Carman K, Dinleyici E. Mycoplasma pneumoniae infection with neurologic complications. Iran J Pediatr 2014;24:647-651.

[5] Panagariya A, Sharma AK, Dev A, Kankane A, Sharma B, Dubey P. Reversible neurological syndromes with atypical pneumonia. Ann Indian Acad Neurol 2011;14:127-129.

[6] Nadkarni N, Lisak RP. GBS with bilateral optic neuritis and central white matter disease. Neurology 1993;43:842-843.

[7] Milla E, Zografos L, Piquet B. Bilateral optic papillitis following Mycoplasma pneumoniae infection. Opthalmologica 1998;212:344-346.

[8] Henderson RD, Ohlrich GD, Pender MP. GBS and optic neuritis after Mycoplasma pneumoniae infection. Aust N Z J Med 1998 Aug;28(4):481-482.

[9] Pfauser B, Englehardt K, Kampfl A, et al. Post-infectious central and peripheral nerve system diseases complicating Mycoplasma pneumoniae infection. Report of three cases and review of the literature. Eur J Neurol 2002;9:93-96.

[10] Candler PM, Dale RC. Three cases of central nervous system complications associated with Mycoplasma pneumoniae. Pediatr Neurol 2004;31:133-138.

[11] Ginestal RC, Plaza JF, Callejo JM, et al. Bilateral optic neuritis and GBS following an acute Mycoplasma pneumoniae infection. J Neurol 2004;251:767-768.

[12] Watanbe $\mathrm{T}$, Hatta $\mathrm{K}$, Ota $\mathrm{T}$, et al. Central nervous system disease complicating Mycoplasma pneumoniae. Psychiatry Clin Neurosci 2007;61:577.

[13] Bae JW, Kim HJ, Chang GY, et al. Combined striatum, brain stem and optic nerve involvement due to 
Mycoplasma involvement in an ambulatory child. Case Rep Neurol 2011;3:109-112.

[14] Benedetti L, Franciotta D, Beronio A, et al. Meningoencephalitis-like onset of post-infectious AQP4IgG-positive optic neuritis complicated by GM1-IgG-positive acute polyneuropathy. Mult Scler 2015;21:246-248.

[15] Chiang WY, Huang HM. Bilateral monosymptomatic optic neuritis following Mycoplasma infection: a case report and literature review. Indian $J$ Ophthalmol 2014;62:724-727.

[16] Ozkale Y, Erol I, Coban-Karatas M. Optic neuritis as a presenting symptom of Mycoplasma infection. Turk J Pediatr 2015;57:401-406.
[17] Narita M. Pathogenesis of extra-pulmonary manifestations of Mycoplasma pneumoniae infection with special reference to pneumonia. J Infect Chemother 2010;16:162-169.

[18] Al-Zaidy SA, MacGregor D, Mahant S, Richardson SE, Bitnun A. Neurological complications of PCRproven $M$. pneumoniae infections in children: prodromal illness duration may reflect pathogenic mechanism. Clin Infect Dis 2015;61:1092-1098.

[19] Ginestal RC, Plaza JF, Callejo JM, Rodríguez-Espinosa N, Fernández-Ruiz LC, Masjuán J. Bilateral optic neuritis and Guillain-Barré syndrome following an acute Mycoplasma pneumoniae infection. J Neurol 2004;251:767-768. 